\title{
Switching of exciton character in double InGaN/GaN quantum wells
}

\author{
T. Suski, ${ }^{1}$ G. Staszczak, ${ }^{1}$ K. P. Korona, ${ }^{2}$ P. Lefebvre, ${ }^{3}$ E. Monroy, ${ }^{4}$ P. A. Drozdz,${ }^{1,2}$ G. Muzioł, ${ }^{1}$ C. Skierbiszewski, ${ }^{1,5}$ \\ M. Kulczykowski, ${ }^{6}$ M. Matuszewski, ${ }^{6}$ E. Grzanka, ${ }^{1,5}$ S. Grzanka, ${ }^{1,5}$ K. Pieniak, ${ }^{1}$ K. Gibasiewicz, ${ }^{1}$ \\ A. Khachapuridze, ${ }^{1}$ J. Smalc-Koziorowska, ${ }^{1,5}$ L. Marona, ${ }^{1,5}$ and P. Perlin ${ }^{1,5}$ \\ ${ }^{1}$ Institute of High Pressures Physics, UNIPRESS, 01-142 Warsaw, Poland \\ ${ }^{2}$ Institute of Experimental Physics, Warsaw University, 02-093 Warsaw, Poland \\ ${ }^{3}$ Laboratoire Charles Coulomb (L2C), University of Montpellier, Centre National de la Recherche Scientifique, Montpellier, France \\ ${ }^{4}$ Université Grenoble-Alpes, CEA-INAC-PHELIQS, 17 Avenue des Martyrs, 38000 Grenoble, France \\ ${ }^{5}$ TopGaN, Sokolowska 29-37, PL-01142 Warsaw, Poland \\ ${ }^{6}$ Institute of Physics, Polish Academy of Sciences, 02-668 Warsaw, Poland
}

(Received 25 April 2018; revised manuscript received 1 August 2018; published 4 October 2018)

\begin{abstract}
The dependence on exciton density of the interwell coupling scheme in a series of InGaN/GaN symmetrical double quantum wells (DQWs) with varying central barrier width was observed. Continuous-wave photoluminescence (cw-PL) and time-resolved photoluminescence (TRPL), measured at low temperature (6-13 K), allowed us to examine the competition between three optical recombination channels, namely, the recombination of (i) intrawell excitons (DXs), (ii) interwell indirect excitons (IXs), and (iii) presumably coupled well excitons (CWXs), built of electron and hole wave functions that are widely spread over the entire DQW structure. We demonstrate a rather abrupt switching effect that relies on the actual exciton density in the system. In cw-PL experiments as a function of the pumping laser power, this switching is characterized by a threshold laser power density above which we observe (i) a significant change of slope of both the power-dependent blueshift and intensity of the ground-state exciton and (ii) the appearance of higher-energy optical emissions. In TRPL, as the PL intensity decays with time, both these effects are visible but in opposite direction, including the PL intensity transfer from the higher-energy state to the ground state. The observed switching is assigned to a change of the dominant excitonic recombination regime: at low pumping densities the dominant emission arises from the extremely long-lived IX, whereas above threshold the dominant emission corresponds to DXs or CWXs, depending on the barrier width. The threshold power density (or threshold time for TRPL) presents a clearly exponential dependence upon the width of the central barrier, which demonstrates the role of carrier tunneling in the overall switching process. The comparison of IXs in nitride QWs with bias electric-field induced IXs in GaAs/AlGaAs DQWs shows that the spectral blueshifts are a few times larger in the former structures. This enhancement originates from the built-in electric field in group-III nitrides, which has truly microscopic character. Moreover, it is argued that the presence of the potential fluctuations due to alloy disorder and the fluctuations of the coupling barrier width have only a secondary effect on the observed switching.
\end{abstract}

DOI: 10.1103/PhysRevB.98.165302

\section{INTRODUCTION}

In recent years, indirect excitons (IXs) in the $\mathrm{GaAs} / \mathrm{AlGaAs}$ semiconductor system have emerged as highly interesting objects for fundamental studies like Bose-Einstein condensation [1-4], but also as the basis for the design of excitonic devices [5-7].

A coupled quantum well (CQW) system is formed by two either identical or different QWs separated by a potential barrier narrow enough to allow carrier tunneling. In such a case, the wave functions originating from one QW extend into the other QW, which means that the electron or hole lies in both QWs simultaneously, but with different quantum probabilities.

In QW systems, optical emission is usually due to the recombination of excitons, i.e., electron-hole (e-h) pairs bound by Coulomb attraction. In the case of identical QWs (Fig. 1), if the barrier between the wells is wide enough [Fig. 1(a)], an exciton can only be formed by an electron and a hole within the same well, and we will therefore call it a direct exciton (DX). If the barrier is thin enough to create a CQW system, two situations can occur, depending on the general band profile of the structure [Figs. 1(b) and 1(c)]. If the overall profile of the CQWs is "flat" (no electric field applied), the resonance between the coupled wells induces the splitting of the ground states of electron and hole into the symmetric and antisymmetric combinations. In that case, the Coulomb attraction binds an electron and a hole having wave functions extended over the entire structure [Fig. 1(b)], constituting what we will call a coupled well exciton (CWX).

If the coupled wells are set out of resonance by, say, an electric field [Fig. 1(c)], then the Coulomb attraction binds an electron confined in one QW with a hole confined in the other QW: the resulting exciton is called a spatially indirect exciton, or simply an IX. In this case, there still remains the possibility of optical transitions involving electron and hole states in the same well, namely, DXs [Fig. 1(c)]. 


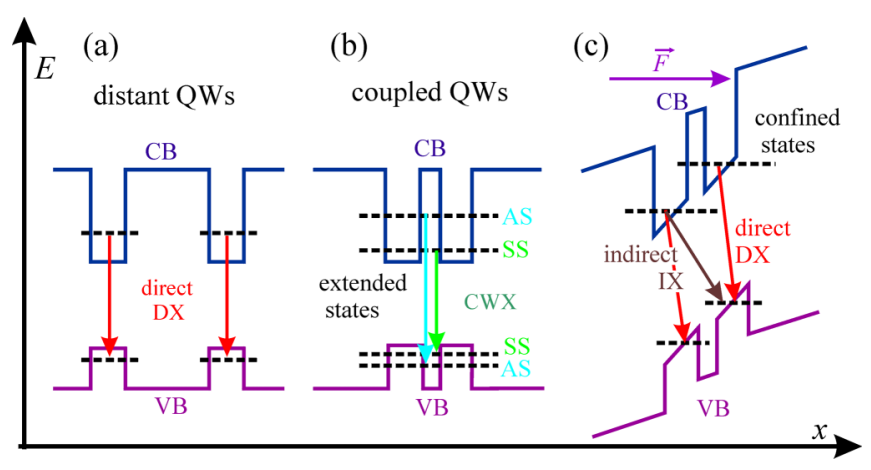

FIG. 1. Definition of excitons in double quantum wells: (a) distant QWs, (b) coupled QWs, and (c) coupled QWs in electric field, $F$. Abbreviations SS and AS correspond to symmetric and antisymmetric extended states, respectively.

In the widely studied CQW structures based on cubic semiconductors (mainly GaAs/AlGaAs), IXs were formed by applying an external electric field perpendicular to the QW planes (see, e.g., Refs. [8-10]). However, due to the relatively small exciton binding energy in arsenide compounds, the practical realization of excitonic devices operating at room temperature seems to be questionable.

The binding energy of excitons in $\mathrm{InN}, \mathrm{GaN}$, and AlN binary compounds is about 20, 30, and 60-70 meV, respectively [11-13]. Therefore, due to the large exciton binding energy, heterostructures such as GaN/AlGaN and InGaN/GaN are much more suitable candidates than arsenide QWs for the study of IXs. Excitons in nitride QWs can be stable over a wide temperature range (even $>100 \mathrm{~K}$ ), when created by excitation with a moderate laser power [14]. At high temperature and/or excitation power, excitons dissociate, experiencing Mott transition into e-h plasma. The latter takes place in a gradual way for carrier concentrations higher than about $10^{12} \mathrm{~cm}^{-2}$ in QWs, and temperatures in the range of 4-150 K [15].

On the other hand, polar wurtzite InGaN/GaN and GaN/AlGaN QWs are characterized by large internal electric fields directed along the $c$ (0001) direction [16-22], due to the difference in spontaneous and piezoelectric polarization between the well and the barriers. Due to its differential nature, the electric field has opposite sign in the well and barrier materials. Nevertheless, Fig. 1(c) can be used with the purpose of qualitative description of a CQW structure based on polar nitrides at zero bias. The states of the two adjacent QWs are clearly out of resonance. Both IX and DX excitons coexist, but the dominant transition in photoluminescence (PL) experiments will be the recombination of the ground state, the IX.

Our paper investigates aspects of the physics of CQWs related to the intense polarization fields in group-III nitrides. The built-in electric field in group-III nitride QWs at zero bias is in the range of megavolts per centimeter [23-28], stronger by orders of magnitude than the external electric field applied experimentally to GaAs/AlGaAs QWs to bring the structure out of resonance (in the kilovolt per centimeter range). As a consequence, the redshift of indirect excitons in nitride CQWs can be of a few hundred meV, whereas for GaAs/AlGaAs this shift is below $20 \mathrm{meV}$. Furthermore, in nitrides, the compensation of the electric field to obtain flat-band CQWs can be obtained not only by biasing the structure but also by the screening of the built-in fields by locally introduced carriers and excitons.

Most of the previous exciton studies in polar wurtzite InGaN/GaN and GaN/AlGaN multiple QWs have been performed in structures with relatively thick barriers separating the QWs (see, e.g., Refs. [12,13,21-27]). Therefore, the observations concerned excitons formed within individual QWs. Nevertheless, it should be noted that, in the case of wideenough single QWs ( $>3 \mathrm{~nm}$, typically), the strong built-in electric field in such QWs causes a significant quantumconfined Stark effect (QCSE) [18,20]. The QCSE leads to the separation of electron and hole wave functions, decreases the emission energy, increases the radiative lifetime [22,24,25] up to several tens or hundreds of microseconds, and produces what was called "naturally indirect" excitons [26,27], with strong permanent dipole moments along the QW growth axis.

Some consequences of this dipolar character in AlGaN/GaN QWs have been reported very recently, with IXs formed in GaN QWs with a width of 6-8 nm, containing or not a one-monolayer-thick AlGaN barrier [26,27]. The intrinsic dipole moment caused in nitride QWs by the large QCSE induces a sufficient repulsive force between IXs to drive their effective transport along the QW plane over large distances (tens of micrometers) and up to high temperatures. The extremely long decay times of those IXs are a crucial ingredient for such long-range transport, boding well for the future realization of condensates of strongly interacting excitons in designed traps. With this perspective, the main aim of the work in Ref. [26] was a comparison of the exciton transport in identical AlGaN/GaN structures grown on sapphire and on bulk GaN substrates. Due to the drastic reduction of defect density achieved in the latter structures, and the very long exciton lifetimes, it was possible to demonstrate exciton transport up to room temperature [26].

On the other hand, if the nitride QW is narrow enough (e.g., $2.6 \mathrm{~nm})$, the field-induced $\mathrm{e}-\mathrm{h}$ separation remains relatively small. Therefore, in the following, whenever an intrawell exciton is encountered, we consider it as a DX. In the present paper, we use description "IX" only for e-h separated by barriers. A crucial characteristic of such IXs is their very long recombination time. One consequence is the possibility, even for moderate laser excitation intensities, to accumulate large densities of IXs, which can lead to the screening of the built-in field. Asymptotically, the system can switch from an outof-resonant situation, where IXs are the dominant transition, to a near flat-band configuration very similar to Fig. 1(b), dominated by recombination of CWXs.

In this paper, we have exploited the possibility of controlling the exciton character in InGaN/GaN CQWs. In order to produce IXs with varying characteristics, we grew double InGaN/GaN QWs with different barrier widths. We could therefore examine the conditions for the practical realization of coupling between two identical InGaN/GaN QWs, by use of low-temperature continuous-wave PL (cw-PL) and time-resolved PL (TRPL) measurements. In these samples, similarly to GaN/AlGaN QWs, large internal electric fields are caused by spontaneous and piezoelectric polarization. We could determine the critical central barrier width above which 
TABLE I. Geometrical characteristics of the QW samples used in this paper.

\begin{tabular}{lccc}
\hline \hline Sample & $\begin{array}{c}\text { QW width } \\
(\mathrm{nm})\end{array}$ & $\begin{array}{c}\text { Number } \\
\text { of QWs }\end{array}$ & $\begin{array}{c}\text { Barrier width } \\
(\mathrm{nm})\end{array}$ \\
\hline A & 2.6 & 1 & \\
B & 5.2 & 1 & 4.16 \\
C1 & 2.6 & 2 & 2.08 \\
C2 & 2.6 & 2 & 1.04 \\
C3 & 2.6 & 2 & 0.78 \\
C4 & 2.6 & 2 & 0.52 \\
C5 & 2.6 & 2 & $0.26-0.52$ \\
C6 & 2.6 & 2 & \\
\hline \hline
\end{tabular}

the QWs can be considered uncoupled, thus inducing only DX recombination. Then, in CQWs, we addressed the abovementioned possibility of screening the internal electric fields by varying the laser power density in cw-PL experiments. As a result, we could observe and analyze in detail the switching between the regimes dominated, respectively, by IX and CWX recombination. In the case of TRPL, the evolution of the PL spectra with time after the excitation laser pulse describes the continuous, time-dependent reduction of the screening of the internal electric field [25].

When working with InGaN QWs, it is important to take into account the effect of the potential fluctuations usually present in such structures $[12,13,21]$. In our paper, potential fluctuations can be caused by both alloy disorder and by a variation of the width of the coupling barrier. In our operation conditions, these fluctuations are expected to be widely saturated by optically injected carriers. In this paper, we discuss the validity of this assumption and calculate the magnitude and potential effect of potential fluctuations in our samples.

\section{SAMPLES AND METHODS}

The set of samples studied in this paper consists of eight structures grown by plasma assisted molecular-beam epitaxy (MBE). Their respective characteristics are gathered in Table I. They were all deposited on bulk GaN substrates with a 2- $\mu$ m-thick GaN:Si buffer layer and a $40-\mathrm{nm} \mathrm{In}_{0.02} \mathrm{Ga}_{0.98} \mathrm{~N}$ buffer layer. Each sample comprises either single [Fig. 2(a)] or double [Fig. 2(b)] $\operatorname{In}_{0.17} \mathrm{Ga}_{0.83} \mathrm{~N}$ QWs. The targeted width of all QWs was $2.6 \mathrm{~nm}$, except for sample B (5.2-nm width). (a)

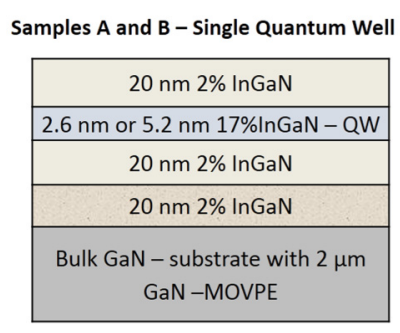

(b) Samples C1-C6 - Double Quantum Well

\begin{tabular}{|c|}
\hline $20 \mathrm{~nm} 2 \% \operatorname{InGaN}$ \\
\hline $2.6 \mathrm{~nm} 17 \% \operatorname{lnGaN}-\mathrm{QW}$ \\
\hline $2 \% \operatorname{lnGaN}-\mathrm{QB}$ \\
\hline $2.6 \mathrm{~nm} 17 \% \operatorname{lnGaN}-\mathrm{QW}$ \\
\hline $20 \mathrm{~nm} 2 \% \operatorname{InGaN}$ \\
\hline $20 \mathrm{~nm} 2 \% \operatorname{lnGaN}$ \\
\hline $\begin{array}{c}\text { Bulk GaN }- \text { substrate with } 2 \mu \mathrm{m} \\
\text { GaN -MOVPE }\end{array}$ \\
\hline
\end{tabular}

FIG. 2. Sketches of the studied $\operatorname{In}_{0.17} \mathrm{Ga}_{0.83} \mathrm{~N} / \mathrm{In}_{0.02} \mathrm{Ga}_{0.98} \mathrm{~N}$ QWs of this paper. (a) Single QWs (samples A and B). (b) Double QWs (samples C1 to C6).
The width of the undoped $\operatorname{In}_{0.02} \mathrm{Ga}_{0.98} \mathrm{~N}$ barriers separating the QWs was varied between 0 and $4 \mathrm{~nm}$ and all samples were capped by 20 -nm-thick undoped $\mathrm{In}_{0.17} \mathrm{Ga}_{0.83} \mathrm{~N}$ barriers. Introducing a small amount of indium into the barriers results in much higher structural quality of interfaces between barriers and QWs in comparison to GaN barriers.

After the growth, the samples were analyzed by highresolution x-ray diffraction (XRD), transmission electron microscopy (TEM), continuous-wave photoluminescence, and time-resolved photoluminescence. The XRD and TEM measurements showed that, with an accuracy of $1 \mathrm{ML}(0.26 \mathrm{~nm})$ and $\pm 1 \%$ of In content, the parameters of both QWs and barriers separating them agreed with the targeted values.

It is worth noting that sample C5 was grown intentionally with a central barrier of two atomic monolayers $(2$ MLs $\approx$ $0.52 \mathrm{~nm}$ ), whereas the targeted barrier width for sample C6 was $1 \mathrm{ML}$. However, TEM studies revealed clear fluctuations of the latter barrier width between 0.26 and $0.52 \mathrm{~nm}$.

The cw-PL spectra were measured at $13 \mathrm{~K}$ with nonresonant excitation laser Lasos DPSSL (wavelength of $320 \mathrm{~nm}$, $3.87 \mathrm{eV}$ ). A maximum power of $20.4 \mathrm{~mW}$ on the sample surface was used, on an estimated area of $38.5 \mu \mathrm{m}^{2}$, therefore with an estimated maximum power density of around $53 \mathrm{~kW} / \mathrm{cm}^{2}$, i.e., $\sim 0.9 \times 10^{23}$ photons $/\left(\mathrm{s} \mathrm{cm}^{2}\right)$. For tuning the laser power, an attenuator was used, enabling collection of spectra down to a minimum laser power of $0.4 \mu \mathrm{W}\left(1 \mathrm{~W} / \mathrm{cm}^{2}\right.$, i.e., $\sim 0.16 \times 10^{19}$ photons $\left./\left(\mathrm{s} \mathrm{cm}^{2}\right)\right)$. However, depending on the PL intensity, in some samples reliable spectra could be obtained only for higher laser power densities than that of minimum power.

The TRPL of the samples was measured using a Hamamatsu streak camera. The samples were excited by the third harmonic of a pulsed Ti:sapphire laser (selected excitation wavelength was $300 \mathrm{~nm}$ ) with repetition frequencies in the $0.04-80-\mathrm{MHz}$ range, using a pulse energy of $0.2-10 \mathrm{pJ}$, corresponding to an average laser power of $0.02-100 \mu \mathrm{W}$ and time-averaged power density $0.1-500 \mathrm{~W} / \mathrm{cm}^{2}$. Typically for a pulse energy of $1 \mathrm{pJ}$ the energy density is of $5 \mu \mathrm{J} / \mathrm{cm}^{2}$, corresponding to $8 \times 10^{12}$ incident photons $/ \mathrm{cm}^{2} /$ pulse (i.e., $\sim 6 \times 10^{20}$ photons $/\left(\mathrm{s} \mathrm{cm}^{2}\right)$ at $\left.80 \mathrm{MHz}\right)$

The band structure and electronic levels were calculated using the NEXTNANO software [29], with the parameters listed in Table II. Parameters for $\operatorname{In}_{x} \mathrm{Ga}_{1-x} \mathrm{~N}$ ternary alloys are generally obtained by linear interpolation between $\mathrm{GaN}$ and InN. Bowing parameters were only used for the calculation of the band-gap energy ( $b=2.1 \mathrm{eV}$ [30,31], applied to the conduction band) and the spontaneous polarization $(b=$ $-0.038 \mathrm{C} / \mathrm{m}^{2}$ [32]). The Schrödinger-Poison equations were solved self-consistently using the effective mass approximation. Note that excitonic effects are not taken into account in the calculations.

The simulated structure consisted of an $\operatorname{In}_{0.02} \mathrm{Ga}_{0.98} \mathrm{~N}$ matrix containing two 2.6-nm-thick $\operatorname{In}_{0.17} \mathrm{Ga}_{0.83} \mathrm{~N}$ quantum wells separated by an $\mathrm{In}_{0.02} \mathrm{Ga}_{0.98} \mathrm{~N}$ barrier of variable thickness. Everything was considered strained on a $\mathrm{GaN}$ substrate.

A general illustration of the band profiles and quantum states is presented in Fig. 3(a) using the sample with a central barrier of $1 \mathrm{~nm}$ as an example. The lower-energy transition is the indirect transition labeled as IX in the figure. The direct 
TABLE II. Parameters used in the calculation of the band structure.

\begin{tabular}{|c|c|c|c|c|}
\hline \multirow{2}{*}{ Lattice constants (nm) } & $a$ & 0.3189 & 0.3545 & \multirow{2}{*}[32,33]{} \\
\hline & $c$ & 0.5185 & 0.5703 & \\
\hline Spontaneous polarization $\left(\mathrm{C} \mathrm{m}^{-2}\right)$ & $P_{\mathrm{SP}}$ & -0.034 & -0.042 & {$[31,32]$} \\
\hline Piezoelectric constants $\left(\mathrm{C} \mathrm{m}^{-2}\right)$ & $e_{13}$ & -0.49 & -0.57 & {$[16]$} \\
\hline \multirow{3}{*}{ Elastic constants (GPa) } & $C_{12}$ & 145 & 115 & \multirow{3}{*}[33,34,35]{} \\
\hline & $C_{13}$ & 106 & 92 & \\
\hline & $C_{33}$ & 398 & 224 & \\
\hline Band gap (eV) & $E_{\mathrm{G}}$ & 3.51 & 0.69 & {$[30,33]$} \\
\hline Conduction-band offset with GaN (eV) & $\Delta E_{\mathrm{CB}}$ & 0 & 2.44 & \\
\hline \multirow{7}{*}{$\begin{array}{l}\text { Deformation potentials }(\mathrm{eV}) \\
a_{c}=\text { conduction band } \\
\text { absolute deformation potential } \\
D_{i}=\text { valence band } \\
\text { uniaxial deformation potential }\end{array}$} & $a_{c, c(c \text { axis })}$ & -4.6 & -0.65 & \multirow{7}{*}[32]{} \\
\hline & $D_{1}$ & -1.7 & -3.7 & \\
\hline & $\mathrm{D}_{2}$ & 6.3 & 4.5 & \\
\hline & $D_{3}$ & 8.0 & 8.2 & \\
\hline & $D_{4}$ & -4.0 & -4.1 & \\
\hline & $D_{5}$ & -4.0 & 4.0 & \\
\hline & $D_{6}$ & -5.6 & -5.5 & \\
\hline
\end{tabular}

transition (DX), located at higher energy, will only be observed if the barrier tunneling time is slower (or comparable) to the IX relaxation time. Therefore, for narrow barriers and low excitation density, IX is expected to be the dominant recombination path.

The photogeneration of carriers was simulated by assuming a homogeneous electron density in the structure. Figures 3(a)-3(c) represent the evolution of the band diagram of the structure with a central barrier of $1 \mathrm{~nm}$ with increasing carrier concentration, namely, (a) $n=10^{17} \mathrm{~cm}^{-3}$, (b) $n=3 \times$ $10^{18} \mathrm{~cm}^{-3}$, and (c) $n=10^{21} \mathrm{~cm}^{-3}$. Figure 3(d) describes the evolution of the IX and DX transition energies as a function of the carrier concentration. For higher carrier concentrations, the internal electric field is increasingly screened, the bands become flatter, and the energy difference between DX and IX decreases. Note that the blueshift of IX in Fig. 3(d) is much stronger than that of DX. Therefore, for high excitation power, the proximity in energy of DX and IX and the higher electron density increase the probability of observation of the direct transition.

Finally, Figs. 3(e) and 3(f) illustrate the band diagram under high excitation power (flat-band conditions) for various widths of the central barrier $(0.52,1$, and $4 \mathrm{~nm})$. For narrow barriers, the electron wave functions extend into the neighboring QW. This means that the probability of observing direct transitions resulting from the coupling of the QW states is high. In contrast, in the structure with a central barrier width of $4 \mathrm{~nm}$, the electrons are well localized in their QWs and the tunneling probability is almost zero. It is expected that DX transitions are observed, but they will not involve coupled states as the barrier is too wide for efficient coupling.

\section{EXPERIMENTAL RESULTS}

\section{A. Photoluminescence power dependence}

Figure 4 shows the evolution of cw-PL spectra as a function of laser power density for samples $\mathrm{C} 1$ and C6. The highenergy side of the spectra reveals the near-band-edge PL from the $\mathrm{GaN}$ substrate/buffer layer at about $3.48 \mathrm{eV}$ and from the $\operatorname{In}_{0.02} \mathrm{Ga}_{0.98} \mathrm{~N}$ thick barriers at about $3.3-3.4 \mathrm{eV}$. The PL peaks in the range of $2.4-2.9 \mathrm{eV}$ are related to the $\mathrm{In}_{0.17} \mathrm{Ga}_{0.83} \mathrm{~N}$ CQWs. For sample $\mathrm{C} 1$, increasing the laser intensity just induces a blueshift $(\sim 50 \mathrm{meV})$ of this latter PL line. We therefore associate it with the recombination of the DXs formed in each distant QW. The 50-meV blueshift is induced by the screening of the QCSE in these relatively narrow QWs, and also to the band filling induced by large carrier densities.

For sample C6, the blueshift is much more pronounced (several hundred meV), which is a characteristic of longlived IXs [9,23,26,27], as explained above. Now, contrary to previously studied systems where a simple, steady blueshift of a single PL line was observed, the results in Fig. 4 are more complex. Between 8 and $2000 \mathrm{~W} / \mathrm{cm}^{2}$, the IX line, indeed, exhibits a steady blueshift of $\sim 0.25 \mathrm{eV}$. Then, above $2000 \mathrm{~W} / \mathrm{cm}^{2}$, this blueshift abruptly follows a much lesser slope. Simultaneously, an additional line appears $\sim 0.1 \mathrm{eV}$ above the previous one, and grows in intensity, while adopting nearly the same blueshift. We interpret these observations as the switching, upon accumulation of IXs, from a regime where the two QWs are out of resonance, to a regime where the coupling becomes closer to resonance. The efficient screening of the internal electric field induces a reconstruction of the 

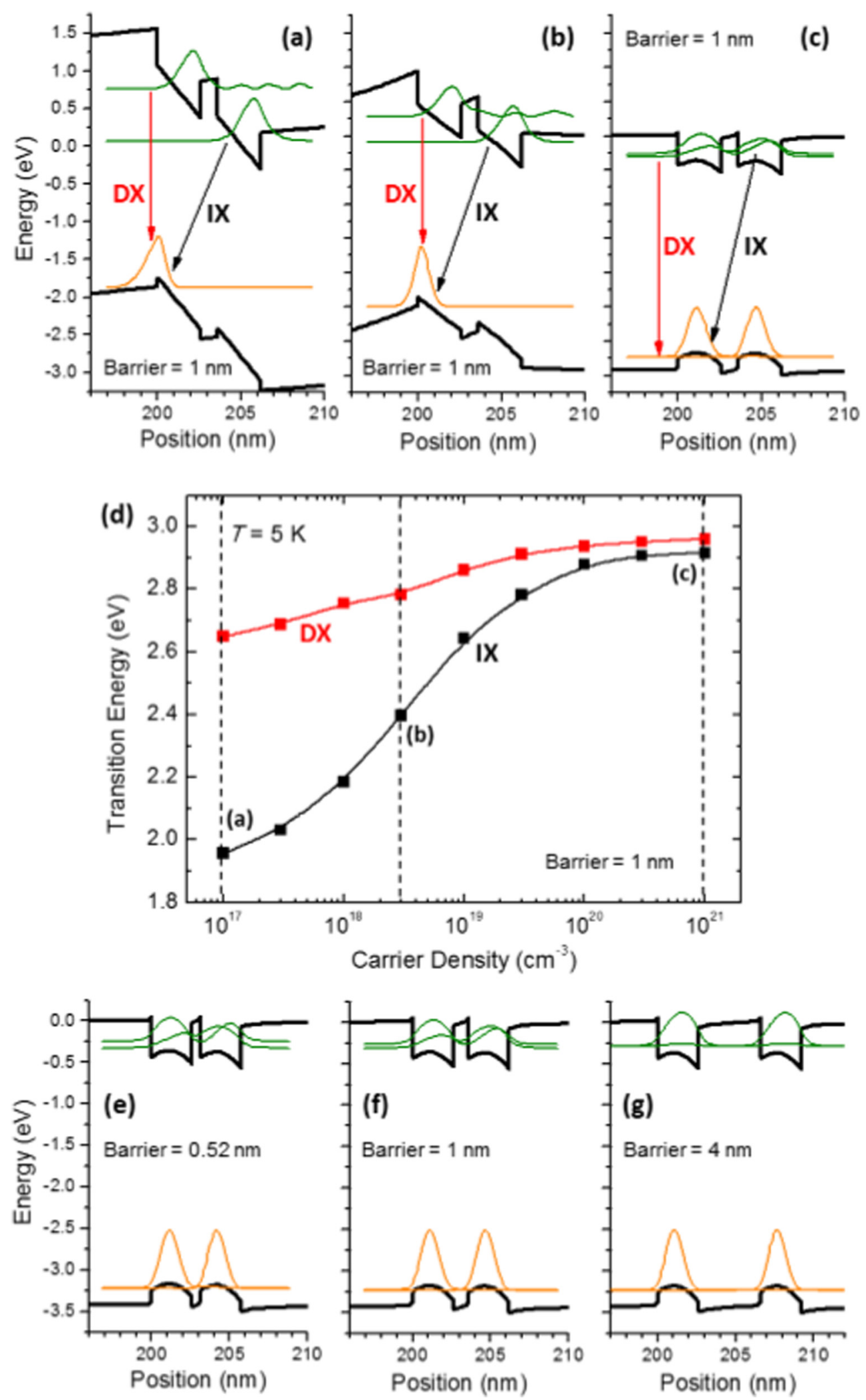

FIG. 3. NEXTNANO modeling of the investigated structures. (a-c) Band diagram of the QWs separated by a barrier of $1 \mathrm{~nm}$ (sample C3) for various carrier densities: (a) $n=10^{17} \mathrm{~cm}^{-3}$, (b) $n=3 \times 10^{18} \mathrm{~cm}^{-3}$, and (c) $n=10^{21} \mathrm{~cm}^{-3}$. The squared wave functions of the main electron and hole levels are represented. Black and red arrows indicate the direct (DX) and indirect (IX) transitions, respectively. (d) Variation of the transition energy of DX and IX as a function of the carrier density in sample C3. Vertical dashed lines mark the points that correspond to the band diagrams in figures (a)-(c). (e-g) Variation of the band diagram of the QWs with high carrier concentration $\left(n=10^{21} \mathrm{~cm}^{-3}\right.$, high enough to fully screen the internal electric field) as a function of the central barrier thickness: (d) $0.52 \mathrm{~nm}$ (sample C5), (e) $1 \mathrm{~nm}$ (sample C3), and (d) 4 nm (sample C1). 

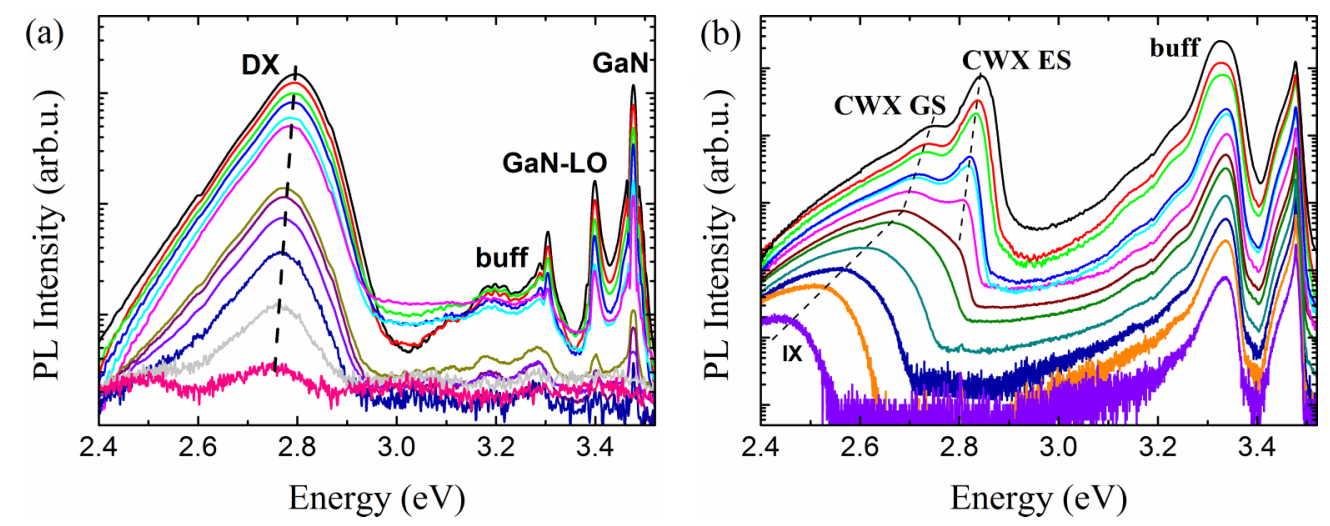

FIG. 4. PL spectra at selected excitation power densities of (a) sample C1 (no IX observed, range of used power densities 5-38000 W/ $/ \mathrm{cm}^{2}$ ) and (b) sample C6, for which IX and CWX states are observed at lower and higher excitation power density, respectively (range of used power densities $0.1-50 \mathrm{~kW} / \mathrm{cm}^{2}$.

overall band profile, accompanied by an efficient "recoupling" of the QWs, therefore inducing quantum states that become extended over the two QWs (CWX). Therefore, above the threshold of $2000 \mathrm{~W} / \mathrm{cm}^{2}$, the IX line transforms into the ground state of the overall CQW system, i.e., into a CWX built upon the symmetric states mentioned above. Obviously, the excited state (ES) that we observe is nothing but the recombination of the CWX built upon the antisymmetric states.

In Fig. 4(b), the reason for the strong blueshift of the emission at low excitation power is the very large recombination time of IXs, which can hence be easily accumulated with moderate excitation intensities, inducing large blueshifts. Now, as soon as the CWX recombination sets up, with quite smaller radiative lifetimes, increasing the excitation power induces less easily the screening of the entire band profile. Due to faster recombination once the CWXs come into play, fewer carriers can be accumulated in the cw regime. Another reason for the reduction of slope of the blueshift is, of course, that once the near-flat-band situation is reached there is no more blueshift to be expected. Note that these results are consistent with our calculations in Fig. 3(d).

The switching in Fig. 4(b) manifests itself not only by spectral changes but also by a sudden change in the dependence of the PL intensity on the laser power density. These sudden changes of (i) emission peak energies, (ii) slopes of blueshifts, and (iii) slopes of intensities versus laser power determine a threshold pumping power density that characterizes the switching process.

Figure 5 summarizes the laser intensity dependencies of PL peak energies for samples A, C1, C3, and C4. Sample A (single 2.6-nm-thick QW) exhibits a weak blueshift with power. This weak and steady blueshift is comparable to the one measured for sample $\mathrm{C} 1$ (DQW with 4-nm-wide barrier). Clearly, for both cases depicted in Fig. 5(a), the observed recombination involves intrawell DXs. The lower PL energy and larger blueshift for sample A are due to a larger internal field in this single QW. Indeed, it is well known that, for multiple QWs, there is an attenuation of the electric field in the QWs with respect to the case of a single well.

Figures 5(b) and 5(c) confirm that, for coupled QWs (narrow barriers), the observations made on samples $\mathrm{C} 3$ and $\mathrm{C} 4$ can be generalized to sample C6. Figures 5(b) and 5(c) clearly show that in samples with sufficiently narrow barriers, at a certain threshold laser power density, $\mathrm{LPD}_{\text {th }}$, the screening of the QCSE by photogenerated carriers leads to the switch from interwell radiative transitions to intrawell transitions. At low temperature, we associate this phenomenon with a change in the exciton character from indirect to direct (IX $\rightarrow$ DX). Moreover, an excited-state transition appears around and above the threshold region. To summarize the information about the $\mathrm{LPD}_{\text {th }}$ dependence on the coupling barrier thickness, we present the evolution of the PL peak energy as a function of the excitation power density for the ground-state transition in Fig. 6. Note that the graph includes single QWs with thicknesses of $2.6 \mathrm{~nm}$ (sample A) and 5.2 $\mathrm{nm}$ (sample B) as extreme situations (infinite coupling barrier and zero coupling barrier, respectively).

In the case of sample B, we observe a change in the slope of the PL energy at an excitation density about $2 \times 10^{3} \mathrm{~W} / \mathrm{cm}^{2}$ (see Fig. 6) together with the emergence of an additional PL line, about $100 \mathrm{meV}$ above the ground state (not shown). This second line can be explained as due to recombination from the second electron state in the QW, which becomes visible due to band filling. This second state is somehow equivalent to the excited antisymmetric state in the CQWs when the coupling barrier tends to zero.

Compiling the results from all samples, we determined the dependence of the $\mathrm{LPD}_{\text {th }}$ on the barrier width (Fig. 7). It is clearly seen that for the barrier widths $\geqslant 0.52 \mathrm{~nm}$ the threshold depends exponentially on the central barrier width. Below this value (sample C6 and sample B), the threshold is constant and equal to $\sim 2 \mathrm{~kW} / \mathrm{cm}^{2}$, suggesting that below 2-ML thickness the barrier plays almost no role.

At the first glance the inspection of Fig. 7 can suggest that the IX-to-DX switch is not observed in the sample with the 4-nm barrier. However, extrapolation of Fig. 7 to the 4-nm barrier allows one to estimate the threshold for switching to $\sim 0.5 \mathrm{~W} \mathrm{~cm}^{-2}$, which is far below the experimental conditions. Moreover, NEXTNANO simulations of the electron-hole wave-function overlap give the negligible value of this entity, which in fact is crucial for enabling the observation of the transition. 

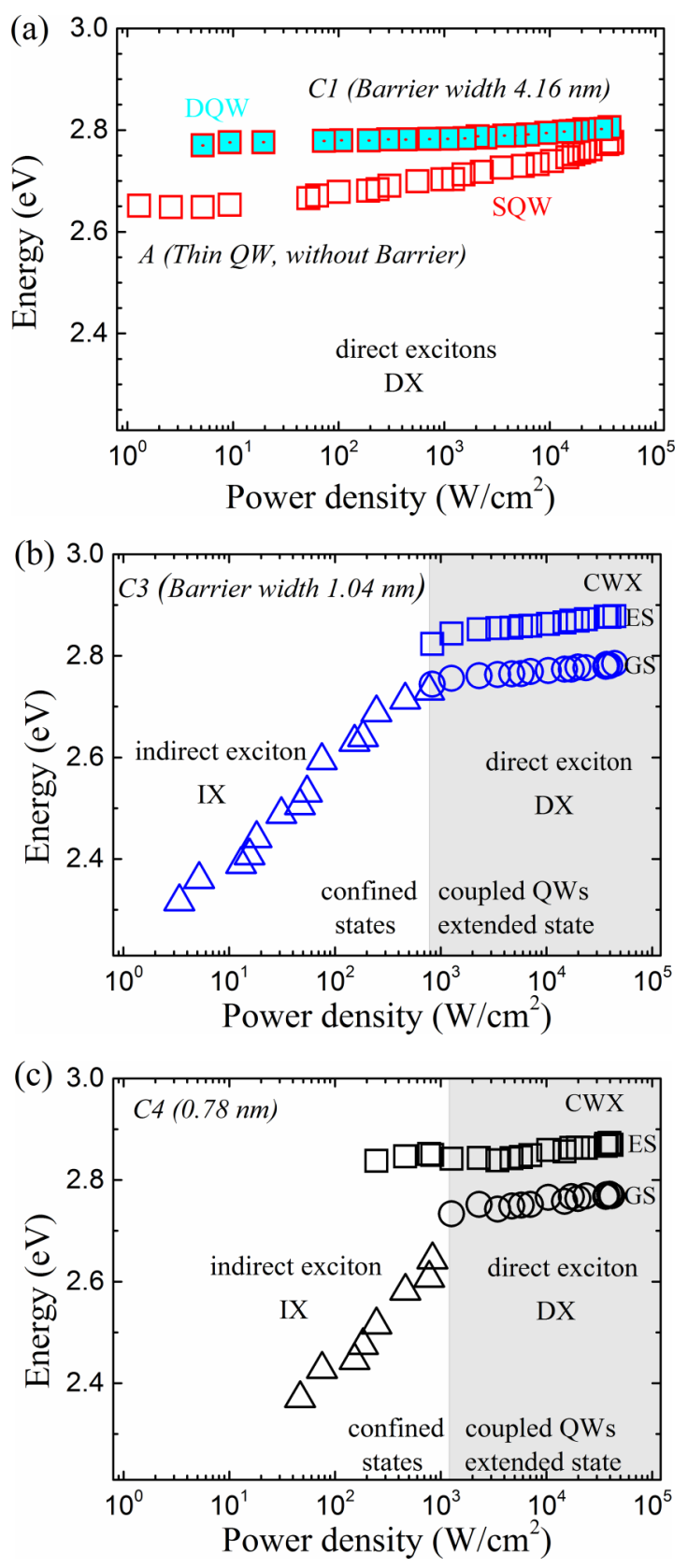

FIG. 5. Dependence of PL energy vs excitation power density for (a) samples A (single QW) and C1 (double QW with 4-nm-wide barrier), (b) sample C3 (double QW with 1.04-nm barrier), and (c) sample C4 (double QW with 0.78-nm barrier).

\section{B. Time-resolved PL}

The TRPL was measured from liquid helium to room temperature. However, most of the TRPL analyses presented here were performed at low temperature of about $6 \mathrm{~K}$. The low-temperature signal from $\mathrm{GaN}$ was at $3.47 \mathrm{eV}$, as expected. Its lifetime was about $0.1 \mathrm{~ns}$, which is significantly faster than in GaN homoepitaxial material $[36,37]$ or pure $\mathrm{GaN}$ nanowires [38]. It was observed that the signal from the CQWs was very strong up to $300 \mathrm{~K}$.

Impulse excitation is characterized usually by timeaveraged power, $P_{\mathrm{av}}$, pulse energy, $E_{\mathrm{p}}$, and frequency, $f$,

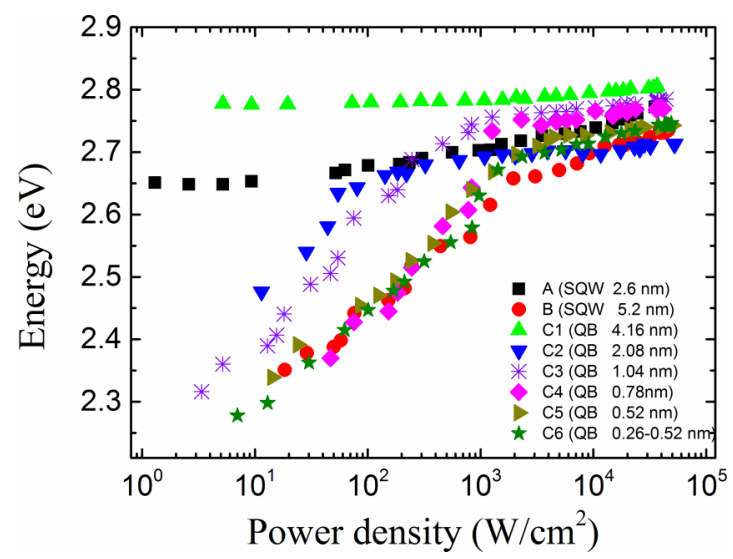

FIG. 6. Evolution of PL energy as a function of excitation laser power density for the excitonic ground states in the studied samples. The single QW samples (SQW) and the width of the quantum barrier (QB) in the DQW samples are indicated in the legend.

where $P_{\mathrm{av}}=E_{\mathrm{p}} f$. For example, pulses of $1 \mathrm{pJ}$ and frequency $1 \mathrm{MHz}$ give $P_{\mathrm{av}}=1 \mu \mathrm{W}$. But, in the case of decay faster than repetition period, the physical effect of $P_{\mathrm{av}}$ cannot be compared with effect of continuous excitation. In order to find the quantity that can be compared some comments should be made. Excitation with power $P$ creates $v=\eta P$ ( $\eta$ denotes efficiency) excitons per second. If effective excitonic lifetime is $\tau_{\text {eff }}$, we can expect $n=v \tau_{\text {eff }}=\eta P \tau_{\text {eff }}$ excitons, which can be compared with $n_{\mathrm{p}}=\eta E_{\mathrm{p}}$ excitons created by pulse. For comparison we assume $n=n_{\mathrm{p}}$ and define the effective power $P_{\text {eff }}=E_{\mathrm{p}} / \tau_{\text {eff }}$. For example, pulses of $1 \mathrm{pJ}$ of frequency $1 \mathrm{MHz}$ give $P_{\text {eff }}=1 \mathrm{~mW}$. It corresponds to the effective power density of $\Phi_{\text {eff }}=5 \mathrm{~kW} / \mathrm{cm}^{2}$ at the excitation spot.

All the studied QWs showed PL peaks in the range of 2.2-2.9 eV. Like in the case of $\mathrm{cw}-\mathrm{PL}$, the increase of power density caused a blueshift of the IX peak, and then the rising of the CWX ES peak.

Figure 8 shows the exemplary case of sample C5, where a strong peak at $2.85 \mathrm{eV}$ dominates the spectrum between 0 and 2 ns after excitation. This peak is observed only at

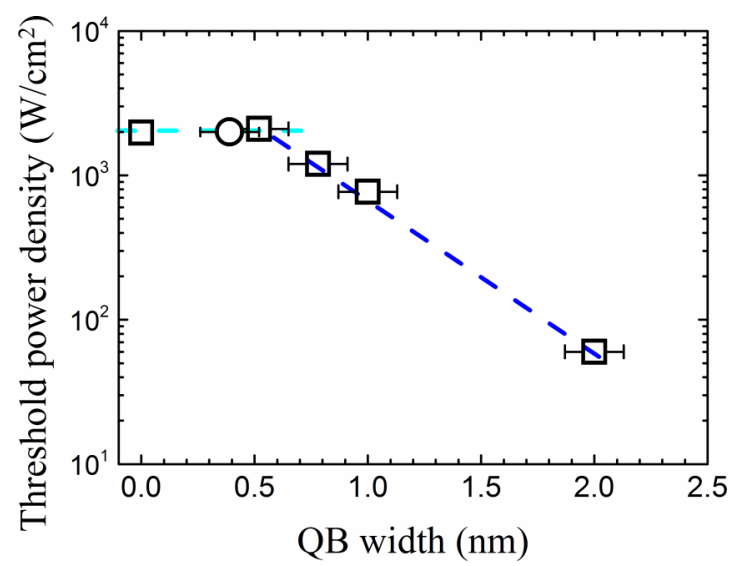

FIG. 7. Variation of threshold power density as a function of the quantum barrier (QB) width. Sample C6 (barrier width = $0.26-0.52 \mathrm{~nm}$ ) is represented by the open black circle at the average barrier width $(0.39 \mathrm{~nm})$. 




FIG. 8. Transient PL spectra of sample C5 (barrier width of $0.52 \mathrm{~nm}$ ) for different time delays after laser excitation, in TRPL measurements. The effective power density was $2.5 \mathrm{~kW} / \mathrm{cm}^{2}$.

high excitation density, which is characteristic of the CWX, as discussed above. The spectra plotted in Fig. 8 were excited with pulses of $0.5 \mathrm{pJ}, \Phi_{\text {eff }}=2.5 \mathrm{~kW} / \mathrm{cm}^{2}$, which is above threshold power density shown in Fig. 7 for continuous excitation. The CWX ES energy slightly decreases with time, as its intensity collapses completely within the first $10 \mathrm{~ns}$ of decay. Then a low-energy peak becomes visible, then dominant, as time passes. We attribute this latter peak to the long-lived IX. Its main characteristic is a large time-dependent redshift, which has been observed quite often in wide-band-gap nitride polar QWs [25-27,37,38]. It is mainly caused by the so-called descreening of the electric field, i.e., by a reestablishment of the QCSE, as the initial high density of photogenerated carriers progressively decreases, by recombination [25,37].

It is important to notice that the spectra plotted in Fig. 8 are spaced in time in logarithmic scale, covering several orders of magnitude ( 0.2 to $400 \mathrm{~ns}$, assuming that the laser pulse excites the sample at time $t=0$ ). In fact, this peak could be measured even several hundred microseconds after excitation. This kind of extremely slow decay is not surprising for IXs in an overall 5.7-nm-wide polar InGaN QW. However, regarding the switching issue, the most important time window in this huge scale is the moment in time when both peaks (IX and CWX ES) show similar magnitudes. For example, for sample C5 (barrier width of $0.5 \mathrm{~nm}$ ) presented in Fig. 8, this time window is about 1-2 ns. For sample C3 (barrier width of $1 \mathrm{~nm})$, this critical time window is rather around 10-20 ns.

The TRPL spectrum of sample C3 is plotted in Fig. 9(a) as a contour map. The contour lines (isolines) in this figure are equally spaced (e-times) in logarithmic scale, so the distance between two contour lines along the time axis indicates an estimation of the decay time of the signal. The decays plotted in Fig. 9(b) have been extracted for spectral windows 2.8-3.0 and 2.6-2.8 eV, so the curves are representative for CWX-ES and IXs, respectively. However, the spectra overlap slightly, so the short time part of the IX curve is obviously influenced by emission from the CWX-ES. It is important to notice that the recombination dynamics changes with time $[25,37,38]$. In such a case, the decay cannot be described by an exponential function that has well-defined decay time. We may, neverthe-
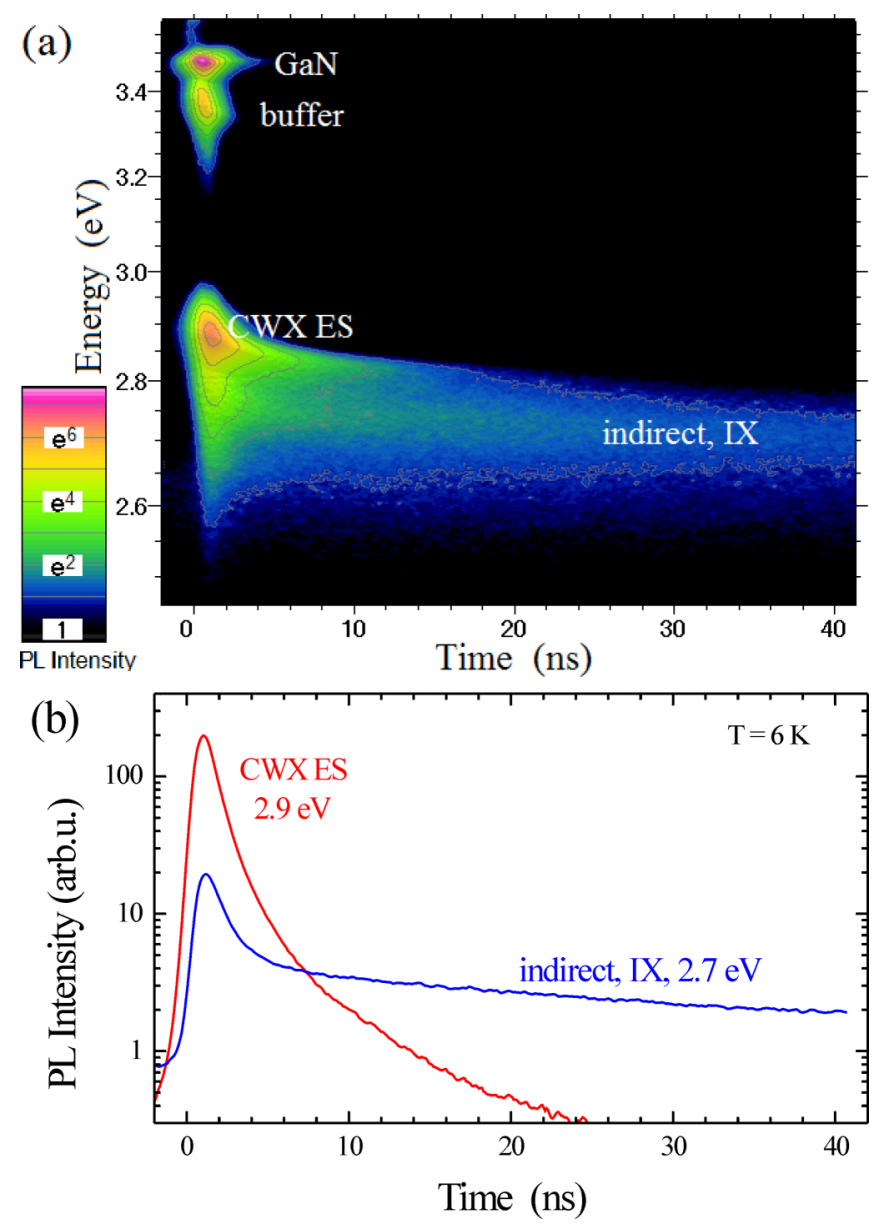

FIG. 9. TRPL of sample C3: (a) full spectrum presented as a contour plot. (b) PL transients observed for CWX- ES and IX (interwell) emissions. The effective power density was $3.5 \mathrm{~kW} / \mathrm{cm}^{2}$.

less, define a time-dependent instantaneous intensity decay rate, $r_{\Phi}$, equal to the logarithmic derivative of the intensity $\Phi(t)$ :

$$
r_{\Phi}(t)=\frac{1}{\Phi(t)} \frac{d \Phi(t)}{d t}
$$

The instantaneous intensity decay time is obtained as $\tau_{\Phi}(t)=1 / r_{\Phi}(t)$. It is worth noting that the electric field changes the oscillator strength of the exciton, so that the intensity decay kinetics is different from the decay kinetics of the exciton concentration. In fact, these two kinetics are strictly identical only in the textbook case of monoexponential decays. Our experiments are extremely far from this case.

The time dependency of the PL energy [Fig. 10(a)] shows many similarities to the power density dependency [Figs. 5(b) and $5(\mathrm{c})]$. This is due to the fact that what really controls the emission energies of the various excitonic emissions is the density of carriers. The latter can be established in a continuous regime of excitation/recombination, and therefore be controlled by the excitation power. In TRPL experiments, this density is simply reached at a given point in time, during the complex decay dynamics. The latter complexity is confirmed by the PL intensity decays, plotted in Fig. 10(b), in log-log scale. The decay time calculated from Eq. (1) changes with 

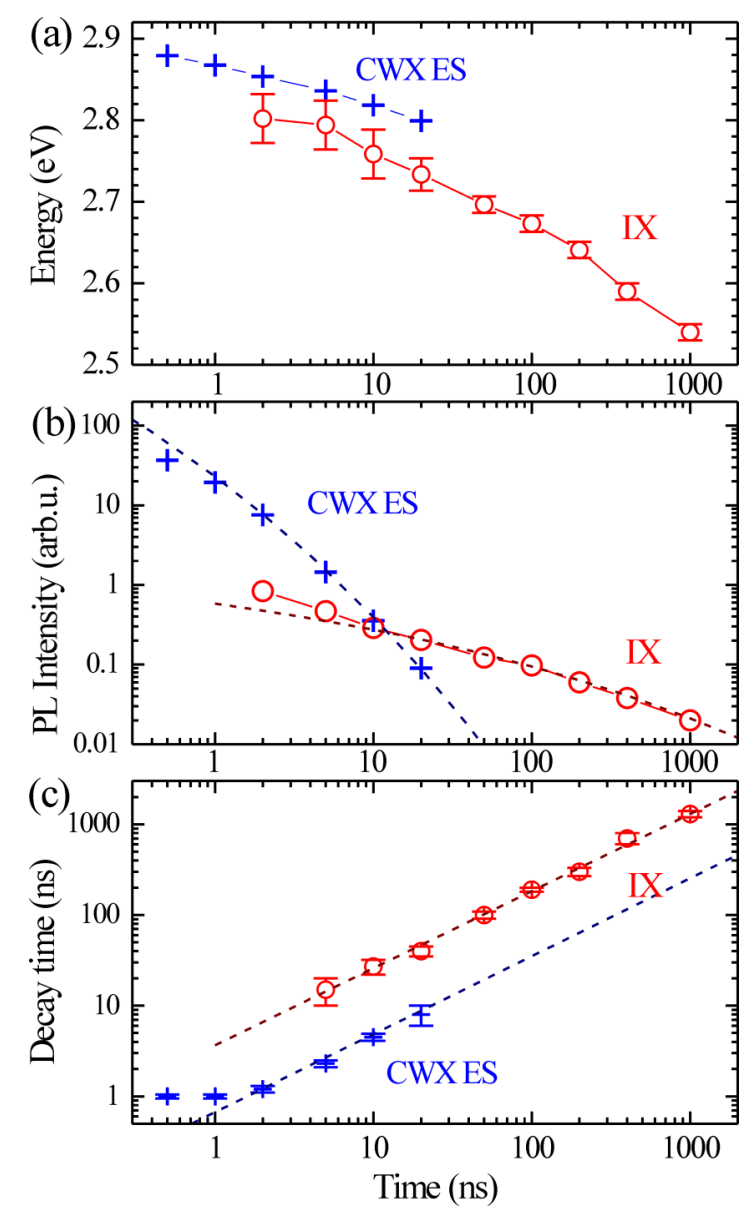

FIG. 10. Temporal evolution of excitonic transitions (IX and CWX-ES) in sample C3: (a) energy, (b) intensity, and (c) instantaneous decay time $1 / r_{\Phi}$.

time. It becomes longer simultaneously with the decrease of intensity and energy, as a result of the reestablishment of QCSE and subsequent drastic reduction of the electron-hole envelope-function overlap. This is precisely the reason why decay time of the IX is longer by orders of magnitude than the CWX ES lifetime.

The experimental intensities and decay times plotted in Figs. 10(b) and 10(c) can be nicely fitted by using a stretched exponential function:

$$
\Phi(t)=M \exp \left[-(t / \theta)^{b}\right] .
$$

The intensity and decay times are correlated by Eq. (1), so that there are only two sets of fitting parameters, one for the ground state (IX) and the second for the excited state (CWX ES). In both sets, the critical exponent was similar: $b=0.15$ and 0.14 for IX and CWX ES, respectively.

Figure 10(b) also shows that the critical time when the PL intensity of CWX-ES becomes smaller than the intensity of the ground IX state is about 10-20 ns for sample C3. This is ten times longer than for sample C5 (barrier width of $0.5 \mathrm{~nm}$ ). It is therefore clear that the switching time between CWX ES and IX transitions strongly depends on the barrier width.

The slowest recombination dynamics that we observed corresponded to the IX in sample C2, i.e., for the CQW with

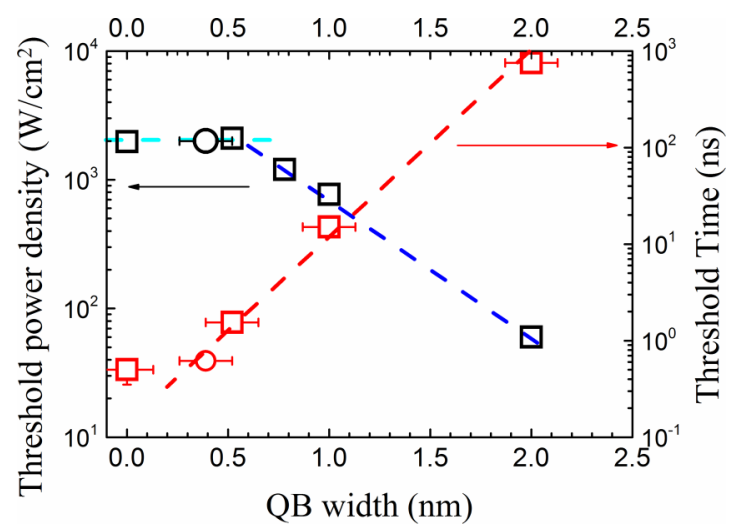

FIG. 11. Illustration of the DX - IX switching as a function of barrier thickness. Open black squares represent threshold power density determined in the cw-PL experiment. Open red square show the threshold time obtained from TRPL. Sample C6 (barrier width $1-2$ atomic monolayer) is represented by the open black and red circles.

central barrier of $2 \mathrm{~nm}$. The lifetime, $\tau_{\Phi}$, that could be calculated from our data reached $100 \mu$ s for this IX. Even for the CWX transitions the lifetime was longer than for thin-barrier samples: it was between 10 and $100 \mathrm{~ns}$. The characteristic time of the CWX ES/IX switching for this sample was about $0.5-1 \mu$ s, i.e., about 50 times longer than for sample C3. The threshold times of CWX ES/IX switching times obtained for the different samples are plotted in Fig. 11.

Comparing TRPL behaviors for samples with different barrier widths we see that the thicker the barrier the longer the excited-state recombination peak (CWX) remains comparable in intensity to the ground-state recombination peak (IX). Moreover, the switching time increases exponentially with the barrier widths (Fig. 11). This supports our previous comment that the switching time (or threshold laser intensity, as seen above) is a matter of competition between the relaxation times of electrons and holes from their excited states, on one hand, and the recombination times of the DX (or CWX), on the other hand. The relaxation times of excited electron and hole states involve the tunneling across the central barrier and it is no surprise that we obtain an exponential dependence on the barrier width for samples with barrier width between 0.39 and $2 \mathrm{~nm}(0.39 \mathrm{~nm}$ refers to average barrier width of sample C6). Sample B (QW of double width with no QB) demonstrates the threshold time similar to sample C6, which recalls the behavior of threshold power density.

\section{Potential fluctuations}

As it has been well established, the potential fluctuations in InGaN/GaN heterostructures are relevant to understand their radiative performance $[12,13]$. However, our structures, due to optimized and careful growth on bulk GaN substrates, by the MBE method, demonstrate a very high uniformity and sharp QW/barrier interfaces verified by TEM studies (with the above-discussed exception of sample C6). The low-temperature growth of MBE compared with the metal organic vapor phase epitaxy (MOVPE) method used for LED production results in reduced miscibility problems and 


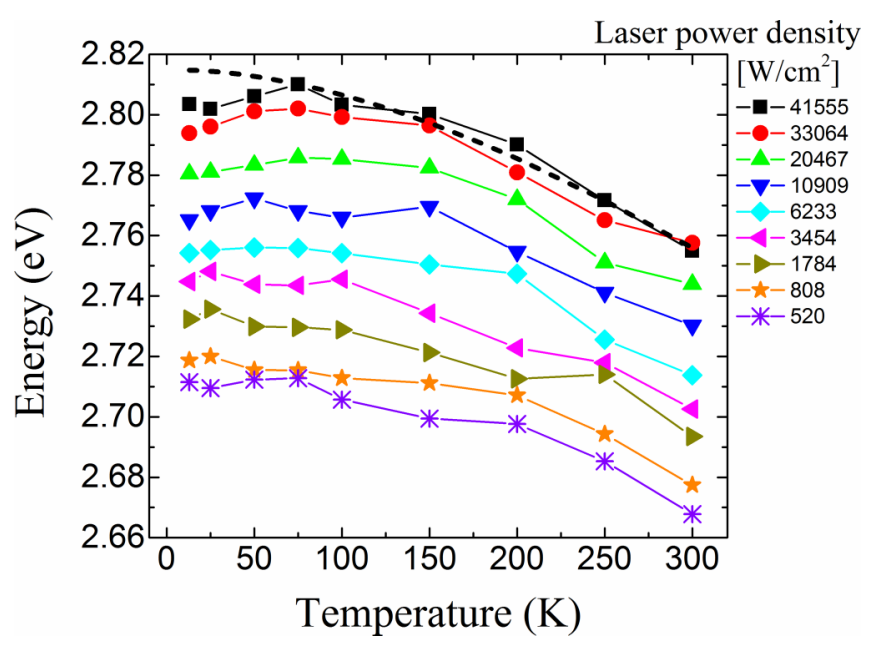

FIG. 12. Evolution of the PL peak position as a function of temperature, measured at various laser power densities. The dashed line describes Varshni's equation, $E_{\mathrm{PL}}(T)=E_{\mathrm{PL}}(T=0)-$ $\alpha T^{2} /(T+\beta)$, with $\alpha=0.590 \mathrm{meV} / \mathrm{K}$ and $\beta=600 \mathrm{~K}$ [39].

sharper heterointerfaces. Our samples have relatively narrow PL emission peaks $(60-70 \mathrm{meV})$ and show nearly monotonic energy-temperature dependence (Fig. 12), which is an evidence of reduced potential fluctuations. Under moderate excitation conditions, the presence of significant potential fluctuations causes the so-called $\mathrm{S}$ shape characterizing the temperature dependence of the peak PL energy (see, e.g., [23]). Now, in case of stronger excitation, the population of those fluctuations by excited carriers can lead to the saturation of the corresponding density of states. For high laser power density, the variation of the PL peak energy with temperature is well described by Varshni's equation for GaN [39], as illustrated in Fig. 12. This is a clear indication that our highexcitation experiments take place in the regime of saturation of the fluctuations. In other words, excitons are placed above the so-called mobility edge. For that reason, all the effects that we describe and comment upon do concern two-dimensional systems where the coupling scheme between the wells and the existence of coupled well excitons can, indeed, be discussed in the context of the usual one-dimensional description of the band profiles. Furthermore, at lower laser power densities, the deviation is smaller than $15 \mathrm{meV}$, which points to alloy fluctuations smaller than $1 \%$.

We have tried to quantify the effect of such potential fluctuation in the switching process. Detailed three-dimensional modeling of the fluctuation landscape was presented very recently in the set of papers [40-42]. Using the proposed approach to our structure is at present beyond our capabilities. Instead, we propose a simplified approach which helps to get some assessment of the role of the potential fluctuations in our structures.

In the studied InGaN/GaN wells, fluctuations can be caused by both alloy disorder in the InGaN QWs as well as the variation of the QW/quantum barrier width. Using the NEXTNANO solver, and the model of the $\operatorname{In}_{0.17} \mathrm{Ga}_{0.83} \mathrm{~N} /$ $\mathrm{In}_{0.02} \mathrm{Ga}_{0.98} \mathrm{~N}$ sample with a central barrier of $1 \mathrm{~nm}$ [see results in Figs. 3(a)-3(d)], we analyzed the effect of varying the In content in the QWs by $\pm 1 \%$ (i.e., $\operatorname{In}_{x} \mathrm{Ga}_{1-x} \mathrm{~N}$ with $x=0.17$,
0.16 , and 0.18 ) and the central barrier width by \pm 1 atomic layers (i.e., 4, 3, and 5 monolayers).

The variation of the In content induces a spectral shift of $\pm 75 \mathrm{meV}$ of the IX transition (value at $n=3 \times 10^{18} \mathrm{~cm}^{-3}$ ), which decreases to $\pm 40 \mathrm{meV}$ when flat-band conditions are reached (value at $n=10^{20} \mathrm{~cm}^{-3}$ ). For DX, the theoretical shift (always in the same direction as that of IX) is $\pm 55 \mathrm{meV}$ at $n=3 \times 10^{18} \mathrm{~cm}^{-3}$, decreasing to $\pm 30 \mathrm{meV}$ at $n=10^{20} \mathrm{~cm}^{-3}$. These shifts are much smaller than the shifts in Figs. 4 and 5, so that our experimental result cannot be explained only by the saturation of the alloy fluctuations. Moreover, in our calculations, the electron-hole wavefunction overlap does not vary by more than +65 to $-40 \%$, i.e., not even a factor of 2 , to be compared with the shift of the PL decay time by several orders of magnitude described in Fig. 10(c). We therefore conclude that alloy fluctuations play only a secondary role in our experiments.

Regarding the effect of monolayer fluctuations of the width of the central barrier, the effect on the energy location of IX and DX for the barrier of $1 \mathrm{~nm}$ is smaller than \pm 25 and $\pm 5 \mathrm{meV}$, respectively, in all the range of carrier densities under study. The main effect of varying the barrier width is the change in the electron-hole wave-function overlap of the indirect transition, IX, which can vary by a factor of 2 at $n=3 \times 10^{18} \mathrm{~cm}^{-3}$, but the effect decreases for increasing pumping power $\left( \pm 5 \%\right.$ at $\left.n=10^{20} \mathrm{~cm}^{-3}\right)$ and it is negligible for DX.

In conclusion, the role of potential fluctuations related to alloy disorder or variation of the thickness of the coupling barrier is minor in the experiments described in this paper.

\section{TEMPERATURE DEPENDENCE OF SWITCHING EFFECTS}

Our experiments described in Sec. III A were performed at low temperatures. They give a clear picture of the physical phenomenon of switching from indirect to direct excitons. A very strong blueshift of $E_{\mathrm{PL}}$ below the critical magnitude of laser power density is observed even at room temperature. At temperatures higher than about $100 \mathrm{~K}$, the observed switching likely involves electrostatically uncoupled electron-hole pairs. However, in this paper we did not study the issue of the temperature induced dissociation and stability of indirect and direct excitons.

At temperatures above the hypothetical Mott transition, the critical laser power density for the indirect-to-direct transition moves to higher values. This shift is explained by the reduction of the exciton lifetime with increasing temperature, due to the activation of nonradiative recombination paths. As a result, higher laser power density is required to accumulate enough free carriers to efficiently screen the built-in electric field.

\section{CONCLUSIONS}

We have investigated the dependence on exciton density of the interwell coupling scheme in a series of InGaN/GaN symmetrical DQWs, with varying central barrier width. Our studies by cw-PL and TRPL allowed us to examine the competition between three optical recombination channels, namely, the recombination of (i) intrawell excitons, (ii) inter- 
well indirect excitons, and (iii) coupled well excitons, which involve electron and hole wave functions that are widely spread over the entire DQW structure. This competition is controlled by the respective characteristic times, such as the radiative recombination times of the various excitons and the interlevel relaxation times of electrons and holes, which depend on the tunneling across the central barrier. Moreover, owing to the extreme sensitivity of the structures to the carrier density, we could modify, either continuously or temporarily, their overall band profiles, via the screening of internal electric fields. The screening induces a large energy shift of the IX emission with strongly nonclassical overall dynamics (with instantaneous decay rates spanning more than three orders of magnitude as the deexcitation covers time windows between fractions of nanoseconds to microseconds). Ultimately, the screening leads to a change in the very nature of the recombining excitons, evolving from IX to DXs or to CWXs, depending on the width of the coupling barrier. We established the clear exponential dependence of the threshold density or time (respectively, for cw-PL and TRPL) upon the central barrier width, thus demonstrating the role of carrier tunneling in the overall switching process. For wide enough (e.g., 4 $\mathrm{nm}$ ) central barriers, only intrawell excitons (or DXs) were observed.

The comparison of indirect excitons in group-IIInitride DQWs with widely studied bias-induced IXs in
GaAs/AlGaAs DQWs shows a few times higher blueshift of the PL energy in the former structures. This enhancement originates from the much higher built-in electric field in nitrides which has truly microscopic character. Due to the order-of-magnitude higher exciton binding energy in nitrides in comparison with arsenides, one can expect much higher thermal stability of excitons in InGaN/GaN DQWs. The switching effects remain up to room temperatures, although they might be related to the unbound electron-hole pairs.

Finally, it is argued that the contribution of the potential fluctuations due to the alloy disorder and a possible diffused character of the interfaces between the QWs and the coupling barrier have only secondary effects on the observed switching. The described effects, beyond their very interesting fundamental character, are potentially important for concepts of optically controlled excitonic devices.

\section{ACKNOWLEDGMENTS}

This work was supported by the Polish National Science Center, Grants No. 2013/11/B/ST3/04263 and No. 2015/17/B/ST7/04091. We also acknowledge valuable discussions with Dr. Marta Kudrawiec (Wroclaw University of Science and Technology) and Prof. Axel Hoffmann (Technische Universitaet - Berlin).
[1] A. Moskalenko and D. Snoke, Bose-Einstein Condensation of Excitons and Biexcitons (Cambridge University, Cambridge, England, 2005).

[2] L. V. Butov, A. L. Ivanov, A. Imamoglu, P. B. Littlewood, A. A. Shashkin, V. T. Dolgopolov, K. L. Campman, and A. C. Gossard, Phys. Rev. Lett. 86, 5608 (2001).

[3] G. Grosso, J. Graves, A. T. Hammack, A. A. High, L. V. Butov, M. Hanson, and A. C. Gossard, Nat. Photon. 3, 577 (2009).

[4] A. A. High, J. R. Leonard, A. T. Hammack, M. M. Fogler, L. V. Butov, A. V. Kavokin, K. L. Campman, and A. C. Gossard, Nature (London) 483, 584 (2012).

[5] A. G. Winbow, A. T. Hammack, L. V. Butov, and A. C. Gossard, Nano Letters 7, 1349 (2007).

[6] Y. Y. Kuznetsova, M. Remeika, A. A. High, A. T. Hammack, L. V. Butov, M. Hanson, and A. C. Gossard, Opt. Lett. 35, 1587 (2010).

[7] P. Andreakou, S. V. Poltavtsev, J. R. Leonard, E. V. Calman, M. Remeika, Y. Y. Kuznetsova, L. V. Butov, J. Wilkes, M. Hanson, and A. C. Gossard, Appl. Phys. Lett. 104, 091101 (2014).

[8] Y. J. Chen, Emil S. Koteles, B. S. Elman, and C. A. Armiento, Phys. Rev. B 36, 4562 (1987).

[9] S. Charbonneau, M. L. W. Thewalt, Emil S. Koteles, and B. Elman, Phys. Rev. B 38, 6287 (1988); H.-J. Polland, L. Schultheis, J. Kuhl, E. O. Göbel, and C. W. Tu, Phys. Rev. Lett. 55, 2610 (1985).

[10] A. Alexandrou, J. A. Kash, E. E. Mendez, M. Zachau, J. M. Hong, T. Fukuzawa, and Y. Hase, Phys. Rev. B 42, 9225 (1990).

[11] M. Dvorak, S.-H. Wei, and Z. Wu,. Phys. Rev. Lett., 110, 016402 (2013).
[12] S. Nakamura and S. F. Chichibu, Introduction to Nitride Semiconductor Blue Lasers and Light Emitting Diodes (Taylor \& Francis, London, 2000).

[13] H. Morkoç, Nitride Semiconductors and Devices (SpringerVerlag, Berlin, 1999).

[14] C. Netzel, V. Hoffmann, T. Wernicke, A. Knauer, M. Weyers, M. Kneissl, and N. Szabo, J. Appl. Phys. 107, 033510 (2010).

[15] G. Rossbach, J. Levrat, G. Jacopin, M. Shahmohammadi, J.-F Carlin, J.-D Ganiere, R. Butté, B. Deveaud, and N. Grandjean, Phys. Rev. B 90, 201308(R) (2014).

[16] F. Bernardini, V. Fiorentini, and D. Vanderbilt, Phys. Rev. B 56, R10024 (1997).

[17] O. Ambacher, J. Majewski, C. Miskys, A. Link, M. Hermann, M. Eickhoff, M. Stutzmann, F. Bernardini, V. Fiorentini, V. Tilak, B. Schaff, and L. F. Eastman, J. Phys.: Condens. Matter 14, 3399 (2002).

[18] D. A. B. Miller, D. S. Chemla, T. C. Damen, A. C. Gossard, W. Wiegmann, T. H. Wood, and C. A. Burrus, Phys. Rev. Lett. 53, 2173 (1984).

[19] M. Buongiorno Nardelli, K. Rapcewicz, and J. Bernholc, Appl. Phys. Lett. 71, 3135 (1997).

[20] M. Leroux, N. Grandjean, J. Massies, B. Gil, P. Lefebvre, and P. Bigenwald, Phys. Rev. B 60, 1496 (1999).

[21] S. Chichibu, T. Azuhata, T. Sota, and S. Nakamura, Appl. Phys. Lett. 69, 4188 (1996).

[22] J. Binder, K. P. Korona, A. Wysmołek, M. Kamińska, K. Köhler, L. Kirste, O. Ambacher, M. Zając, and R. Dwiliński, J. Appl. Phys., 114, 223504 (2013). 
[23] Y.-H. Cho, G. H. Gainer, A. J. Fischer, J. J. Song, S. Keller, U. K. Mishra, and S. P. DenBaars, Appl. Phys. Lett. 73, 1370 (1998).

[24] P. Lefebvre, J. Allegre, B. Gil, H. Mathieu, N. Grandjean, M. Leroux, J. Massies, and P. Bigenwald, Phys. Rev. B 59, 15363 (1999).

[25] P. Lefebvre, S. Kalliakos, T. Bretagnon, P. Valvin, T. Taliercio, B. Gil, N. Grandjean, and J. Massies, Phys. Rev. B 69, 035307 (2004).

[26] F. Fedichkin, T. Guillet, P. Valvin, B. Jouault, C. Brimont, T. Bretagnon, L. Lahourcade, N. Grandjean, P. Lefebvre, and M. Vladimirova, Phys. Rev. Applied 6, 014011 (2016).

[27] F. Fedichkin, P. Andreakou, B. Jouault, M. Vladimirova, T. Guillet, C. Brimont, P. Valvin, T. Bretagnon, A. Dussaigne, N. Grandjean, and P. Lefebvre, Phys. Rev. B 91, 205424 (2015).

[28] J. S. Im, H. Kollmer, J. Off, A. Sohmer, F. Scholz, and A. Hangleiter, Phys. Rev. B 57, R9435(R) (1998)..

[29] S. Birner, T. Zibold, T. Andlauer, T. Kubis, M. Sabathil, A. Trellakis, and P. Vogl, IEEE Trans. Electron Devices 54, 2137 (2007).

[30] I. Gorczyca, T. Suski, N. E. Christensen, and A. Svane, Phys. Rev. B 83, 153301 (2011).

[31] V. Fiorentini, F. Bernardini, and O. Ambacher, Appl. Phys. Lett. 80, 1204 (2002).

[32] I. Vurgaftman and J. R. Meyer, J. Appl. Phys. 94, 3675 (2003).
[33] A. Polian, M. Grimsditch, and I. Grzegory, J. Appl. Phys. 79, 3343 (1996).

[34] A. F. Wright, J. Appl. Phys. 82, 2833 (1997).

[35] H. Morkoç, Nitride Semiconductor Devices: Fundamentals and Applications (Wiley, New York, 2013).

[36] J. Borysiuk, K. Sakowski, P. Dróżdż, K. P. Korona, K. Sobczak, G. Muzioł, C. Skierbiszewski, A. Kamińska, and S. Krukowski, J. Appl. Phys. 120, 015702 (2016).

[37] K. P. Korona, J. P. Bergman, B. Monemar, J. M. Baranowski, K. Pakuła, I. Grzegory, and S. Porowski, in Proceedings of the 19th International Conference on Defects in Semiconductors ICDS-19, edited by G. Davies and M. H. Nazaro (Trans Tech Publications Ltd, Switzerland, 1997), Vol. 258-263, pp. 1125-1130.

[38] K. P. Korona, A. Reszka, M. Sobanska, P. S. Perkowska, A. Wysmolek, K. Klosek, and Z. R. Zytkiewicz, J. Luminescence 155, 293 (2014).

[39] Y. Li, Y. Lu, H. Shen, M. Wraback, M. G. Brown, M. Schurman, L. Koszi, and R. A. Stall, Appl. Phys. Lett. 70, 2458 (1997).

[40] M. Filoche, M. Piccardo, Y.-R. Wu, C.-K. Li, C. Weisbuch, and S. Mayboroda, Phys. Rev. B 95, 144204 (2017).

[41] M. Piccardo, C.-K. Li, Y.-R. Wu, J. S. Speck, B. Bonef, R. M. Farrell, M. Filoche, L. Martinelli, J. Peretti, and C. Weisbuch, Phys. Rev. B 95, 144205 (2017).

[42] C.-K. Li, M. Piccardo, L.-S. Lu, S. Mayboroda, L. Martinelli, J. Peretti, J. S. Speck, C. Weisbuch, M. Filoche, and Y.-R. Wu, Phys. Rev. B 95, 144206 (2017). 